\title{
New Perspectives for Magnetic Fluid-Based Devices Using Novel lonic Liquids as Carriers
}

\author{
Laura Rodríguez-Arco, Ana Gómez-Ramírez, Juan D.G. Durán \\ and Modesto T. López-López
}

Additional information is available at the end of the chapter

http://dx.doi.org/10.5772/51398

\section{Introduction}

Magnetic materials, that is to say, materials which respond to external magnetic fields, have attracted great interest since around $5^{\text {th }}$ century BC. The ability of materials such as iron or magnetite to be attracted by permanent magnets has always involved some kind of mystery, although magnetism principles are well understood nowadays, especially after J.C. Maxwell stated the basis of electromagnetism in 1865. Magnetism plays a crucial role in our lives these days, being present in many technological applications surrounding us. Nevertheless, only solid magnetic materials can be found in nature. In the search for new ones that could be used in novel technological applications, scientists in the $18^{\text {th }}$ century tried to prepare field-responsive liquids by dispersing particles of magnetic materials in liquid carriers. Actually, the first attempt can be attributed to G. Knight (1779), who suspended iron filings in water that quickly settled (Popplewell, 1984). The preparation of magnetic fluids has undergone great development since then, and magnetic fluids with particles of different nature, size or shape and a wide range of liquid carriers have been reported in the literature.

In this chapter an overview of magnetic fluids and their applications is made, focusing on the latest developments in the field. More specifically, the use of novel ionic liquids as carrier fluids is described. The interest in doing so lies in the fact that ionic liquids may give rise to a new generation of magnetic fluids with promising technological applications.

\section{Magnetic fluids}

As it has been pointed out above, the term "magnetic fluids" (MFs) is used to describe a group of smart materials whose properties can be controlled by means of external magnetic fields. They all are suspensions of magnetisable particles dispersed in a liquid carrier. 
Basically, two kinds of MFs can be defined according to the size of the dispersed phase: magnetorheological fluids (MRFs) and ferrofluids (FFs).

MRFs consist of micron-sized ferro- or ferrimagnetic particles dispersed in a liquid carrier. Traditionally, organic solvents such as kerosene or mineral oil are used as carriers. With regards to the dispersed particles, they can be considered as multi-domain from the magnetic viewpoint, since their size is much higher than the one of a single magnetic domain for the constituent material in question (Bossis et al., 2002). For example, typical MRFs can be composed of iron particles with diameters around $1 \mu \mathrm{m}$, while the size of one magnetic domain in this material is approximately $30 \mathrm{~nm}$ (Herpin, 1968). As a result, MRFs strongly respond to external magnetic fields, giving rise to considerable changes in their flow (rheological) behaviour. More specifically, they behave as Newtonian fluids in the absence of magnetic field application, while their behaviour becomes that of a nonNewtonian plastic fluid when a magnetic field is applied. In this latter case, the MRF only flows when the shear stress applied to the suspension overcomes a finite value, the so-called yield stress. Therefore, it could be said that their rheological behaviour changes from a liquid-like to a solid-like one due to the application of external fields. This change, known as magnetorheological (MR) effect is a consequence of the formation of magnetically-induced structures by the dispersed particles, that have to be broken by the hydrodynamic forces to make the suspension flow (Bossis et al., 2002). Figure 1 shows typical MRF rheograms (shear stress vs. shear rate curves) both in the absence of and upon magnetic field application; the appearance of the yield stress $\left(\sigma_{y}\right)$ can be clearly observed. The field-induced structures and their evolution as the shear rate is increased are schematized too.

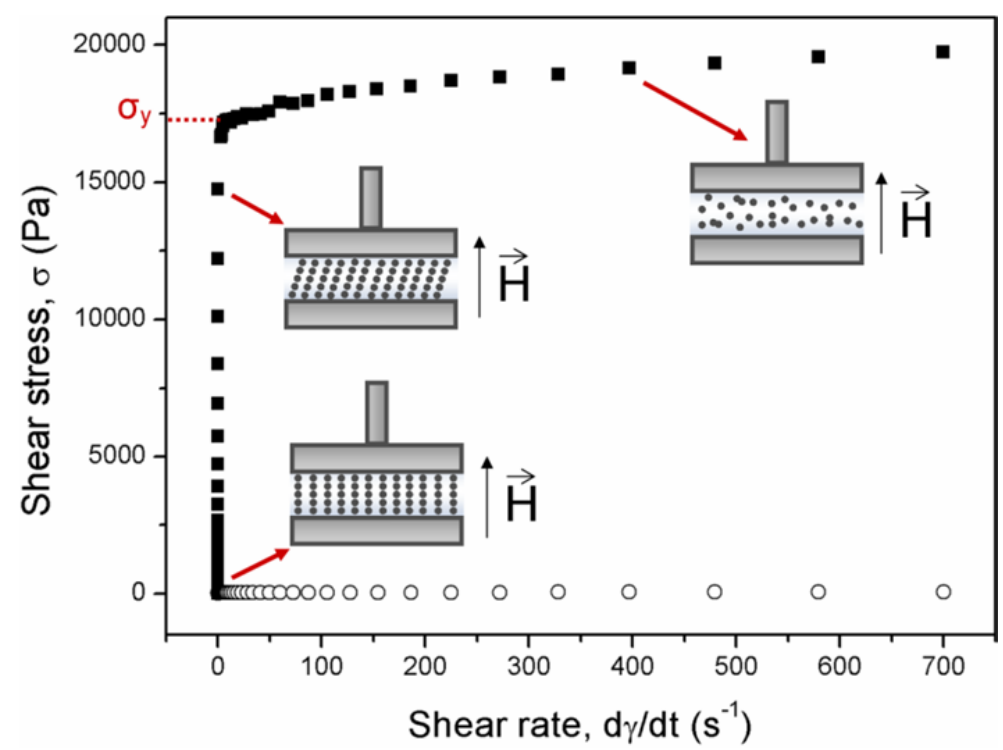

Figure 1. Rheograms for a MRF consisting of $30 \mathrm{vol} \%$ of iron microparticles in mineral oil at magnetic field strengths $(\mathrm{H})$ of 0 and $250 \mathrm{kA} / \mathrm{m}$ (circles and squares respectively). The evolution of the fieldinduced structures is showed. 
The strong magnetic interaction between the dispersed particles in MRFs, responsible for the MR effect, has its disadvantages too. Actually, it makes them agglomerate and settle down if no stabilizing additives are used. Since complete avoidance of sedimentation in MRFs is almost impossible, the efforts in formulation have focused on trying to reduce it and making particle redispersion easy. Different additives such as surfactants (oleic acid, lecithin, aluminum stearate) or thixotropic agents (organoclay particles) have been used for this purpose (de Vicente et al., 2003; López-López et al., 2005a, 2008).

FFs, on the other hand, are ultrastable suspensions of magnetic nanoparticles (size around $10 \mathrm{~nm}$ ) dispersed in a liquid carrier. From the magnetic point of view, these particles are single-domain and therefore, FFs behave as superparamagnetic systems (Charles, 2002; Rosensweig, 1985). The term superparamagnetism is used to describe the magnetic behaviour of systems that exhibit high saturation magnetization values (i.e. typical of ferromagnetic materials), and no magnetic remanence or hysteresis as it happens in paramagnetic materials. FFs only undergo slight changes of viscosity in the presence of external magnetic fields. This phenomenon, known as magnetoviscous (MV) effect is very interesting from the technological viewpoint (Odenbach et al., 2002).

Nevertheless, the most important feature related to FFs is their so-called ultrastability: ideally, true FFs should not settle either when subjected to strong magnetic field gradients or in the presence of gravitational forces during their lifetime (Rosensweig, 1985). As a result, they really behave as magnetic liquids, since no phase separation appears. Figure 2 shows how a FF climbs the tube walls in order to move towards the zones where the magnetic field provided by the magnet is higher.

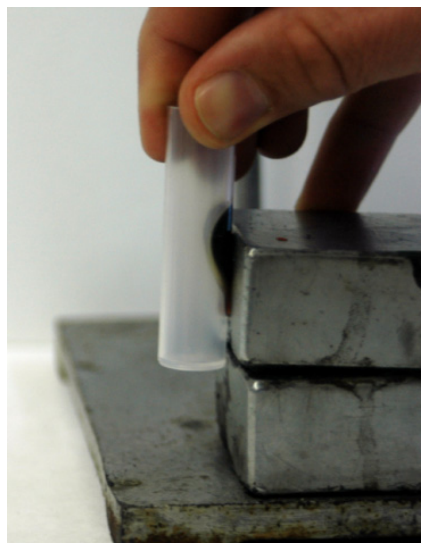

Figure 2. FF ( $5 \mathrm{vol} \%$ of magnetite) subjected to magnetic field application by a powerful rare-earth magnet. It can be seen that the liquid moves as a whole towards the zone where the field is more intense.

Ultrastability in FFs is usually achieved as follows (Charles, 2002; Rosensweig, 1985): (i) particle size is low enough so that Brownian motion prevents from possible particle aggregation induced by the magnetic interaction between the particles; (ii) particle 
aggregation by means of van der Waals attraction (short range forces) must also be avoided by imposing some kind of repulsion between the particles. Such repulsion is usually obtained by adsorbing surfactants or polymers on the surface of the particles, and thus providing with a strong enough barrier (steric repulsion) to overcome van der Waals interactions. In polar media (i.e. water-based FFs), this can be alternatively achieved by electrostatic repulsion between the particles derived from their surface charge (Charles, 2002; Rosensweig, 1985).

The efforts made in order to improve MFs in terms of stability, durability, MR and MV effects have led to the development of a new generation of MFs. This group would include inverse FFs, bimodal suspensions, and MRFs consisting of anisotropic particles. In the first case, non-magnetic microparticles are dispersed in a concentrated enough FF. These particles behave as non-magnetic holes in a practically continuous magnetic medium, and, as a result, are able to orientate and form magnetic structures when a magnetic field is applied (figure 3). This sort of MR effect is stronger than in the case of FFs, but it is still far from that of MRFs (de Gans et al., 1999; Ramos et al., 2011).

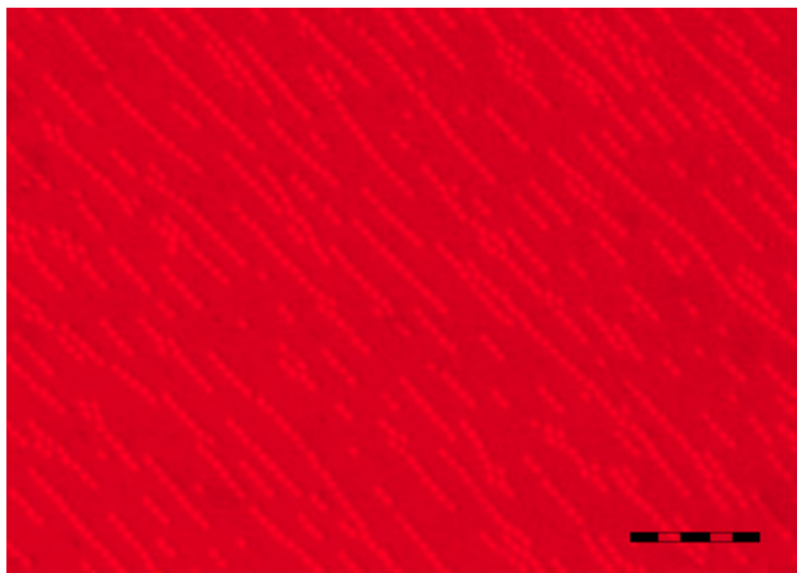

Figure 3. Microscopic picture upon magnetic field application of an inverse FF consisting of PMMA microparticles dispersed in a FF ( $5 \mathrm{vol} \%$ of magnetite in mineral oil). It can be seen that the nonmagnetic particles (white spots) form chains in the direction of the magnetic field. Bar length: $100 \mu \mathrm{m}$.

In the case of bimodal suspensions, micron-sized particles are again dispersed in a FF, but this time, the dispersed particles are magnetic. These MFs have proved to be more stable against sedimentation than conventional MRFs due to the stabilization achieved by the formation of nanoparticle clouds around each micron-sized particle. The formation of such clouds can be observed in figure 4 (López-López et al., 2005b, 2006, 2010).

Finally, the anisotropic nature of fibrillar particles leads to an increase of the MR effect in MRFs consisting of them (Kuzhir et al., 2009; López-López et al., 2007, 2009, 2012). Figure 5 shows magnetic fibrillar particles, with a longitudinal axis of about $50 \mathrm{~nm}$, which have been used in the preparation of stable FFs. 


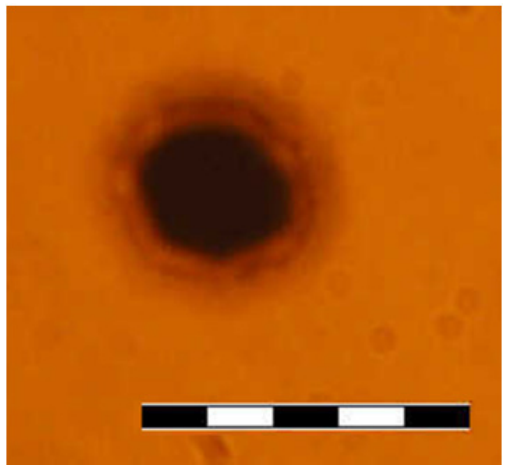

Figure 4. Optical microscope picture of a diluted bimodal suspension consisting of ferromagnetic microparticles dispersed in a FF. Note the halo around each microparticle formed by the magnetite nanoparticles of the FF carrier. Bar length: $20 \mu \mathrm{m}$.

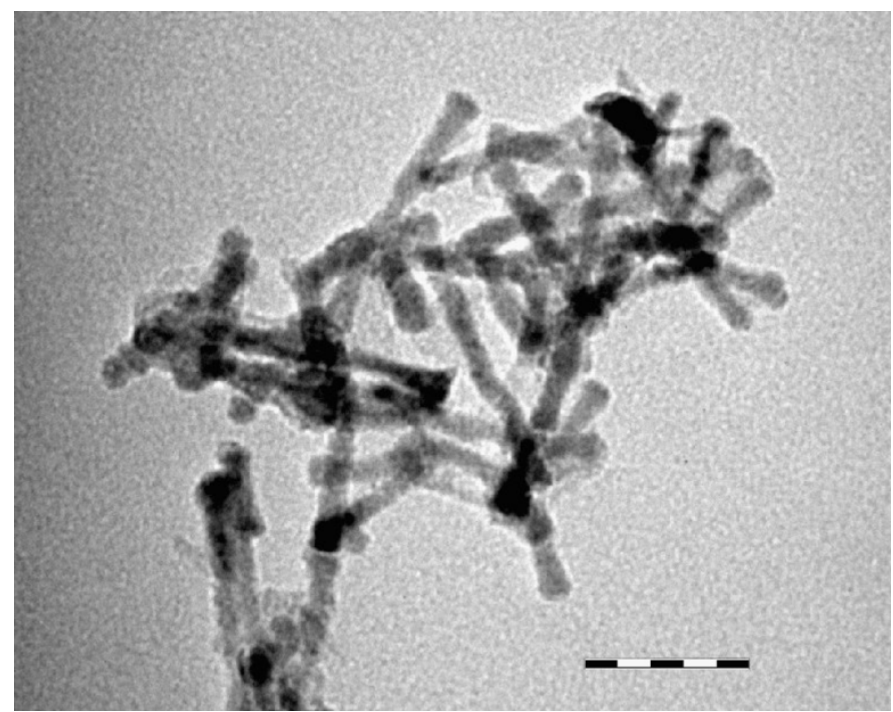

Figure 5. High resolution TEM image of CoNi fibrillar particles for the preparation of MFs. Bar length: $50 \mathrm{~nm}$.

\section{Magnetic fluid-based applications and devices}

The possibility of controlling the physical properties of MFs by external magnetic fields makes them versatile smart materials that can be employed in numerous technological applications, ranging from fields as different as civil and mechanical engineering, aerospace, biomedicine or optics (Carlson et al., 1996; Durán et al., 2007; Jeong et al., 2007; Jolly et al., 1999; Klingenberg, 2001; Park et al., 2010; Popplewell, 1984; Raj et al., 1990; Tran et al., 2010). 
The best known application for MRFs is their use as lubricants with field-dependent viscoelasticity in shock absorbers or dampers. It was in the early 1990s when Lord Corporation $^{\odot}$ started commercializing the first MR dampers. Since then, further progress has followed, and commercial MR dampers have become available for large trucks or race and high-quality cars (Klingenberg, 2001). A typical monotube design of a MR damper used in vehicles was reported by Jolly et al. (Jolly et al., 1999). As shown in this previous work, this kind of MR damper has one reservoir for the MR fluid and an accumulator receptacle full with a compressed gas, which is used to accommodate the volume changes due to the entrance of the piston rod into the housing. When needed, an electric current in the coil generates a magnetic field in the required direction that makes the MRF change its rheological behaviour to a solid-like one; as a result, the vibrations provoked by external forces during driving are absorbed. Therefore, this kind of damping function can be labeled as semi-active (Jolly et al., 1999).

The technological applications of MR dampers are not only restricted to vehicles. For example they are also used as shock/vibration absorbers in structures (i.e. seismic control of buildings or bridges). In this particular case, the stability against sedimentation plays a very important role, since the damper is expected to remain inactive most of its lifetime (Jolly et al., 1999; Klingenberg, 2001; Park et al., 2010). In addition, MR shock absorbers can also be used in semi-active human prosthetic legs.

In the case of FFs, many biomedical applications have been described, which take advantage not only of their superparamagnetic behaviour, but also of their high surface-volume ratio, high reactivity, etc. Medical applications include cell labeling and targeting, separation and purification of cell populations, tissue repair, targeted drug delivery, magnetic resonance imaging or hyperthermia for cancer treatment (Durán et al., 2007; Tran et al., 2010). In all these applications biocompatibility and non-toxicity are of crucial importance. For this reason, iron oxides are preferred as the material for the dispersed phase and, in addition, they are often made biocompatible by means of surface coating by polymers (PEG, dextran, polyvinyl alcohol) or functional groups (thiols, amines, and carboxyls). All these additives prevent from particle aggregation too, which should be almost completely avoided so that particle size does not increase (Tran et al., 2010).

From a more engineering point of view, the control of both the position and the physicochemical properties of FFs by using magnets or solenoids makes them very interesting too. In fact, FFs have been used as lubricants, heat transfer agents or integrated in devices such as dynamic seals, dampers, magnetic inkjets or optic devices. As an example,

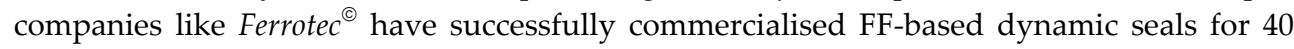
years. Dynamic seals are used when two different environments have to be isolated one from each other but energy has to be carried between them, by a shaft for example (see figure 6). Here, FFs can be used as filling materials, since they hermetically isolate both environments due to their fixed position if the hole for the shaft is made into a magnet. An important advantage of using a seal like this, apart from being almost completely hermetic, is a prolongation of the seal lifetime, since friction between its rotating and stationary components is almost negligible (i.e. the FF acts as a lubricant agent too) (Raj et al., 1990). 


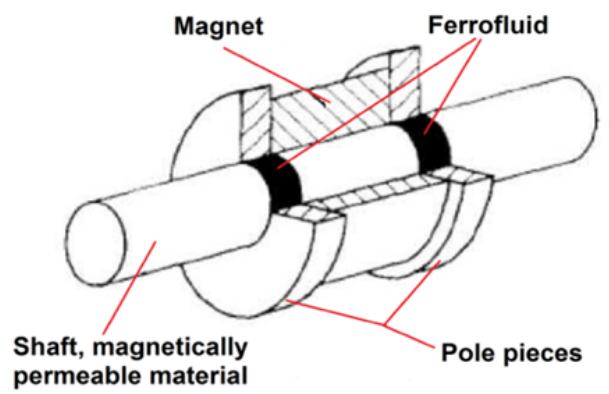

Figure 6. Sketch of a FF-based seal. Reprinted from Journal of Magnetism and Magnetic Materials, 85/13, K. Raj and R. Moskowitz, Commercial applications of ferrofluids, 233-245, Copyright (1990), with permission from Elsevier.

Finally, as it has been pointed out above, FFs can also be used as heat transfer materials, provided that their thermal conductivity increases when applying a magnetic field in a direction parallel to the temperature gradient. In this way, magnetically-induced particle chains, able to transfer heat by conduction, are formed in the field direction. When the field is removed, thermal conductivity reaches its original value (Shima et al., 2011). This working principle is used to dissipate heat from devices such as high power loudspeakers. In this application, the use of FFs has additional advantages like the absorption of undesirable vibrations and avoiding resonances without disturbing the quality of the sound (due to their liquid condition), or the possibility of fixing their position by magnets (as it happens in dynamic seals) (Popplewell, 1984; Raj et al., 1990).

All the applications mentioned in this section are quite well known, and some of them have already been developed at the industrial scale. However, it is quite clear that the range of technological applications of MFs will expand as progress in their dynamics and preparation is made.

\section{Ionic liquids as carriers for magnetic fluids}

Traditionally, organic carriers (mineral oil, kerosene, etc.) have been used in the preparation of MFs, although in some cases (i.e. medical applications) water-based MFs are needed too. These conventional MFs have proved to be useful for many applications as pointed out above. However, the use of novel ionic liquids (ILs) as carriers may promote further improvements in the performance of MFs, especially in ultra-high vacuum applications, in which organic solvents would easily evaporate. But the preparation of IL-based MFs is not only interesting from an applied, technological approach, but also from the fundamental one. For example, their use as carriers allows studying phenomena involving MFs in environmental conditions that conventional carriers would not withstand. But, what are ILs and why do they appear as promising candidates in the preparation of MFs? These and other questions are discussed in next sections. 


\subsection{Ionic liquids}

ILs are substances composed entirely of ions that are liquids at room temperature (Endes et al., 2008). While common salts, such as table salt (i.e. sodium chloride), have melting points around $800{ }^{\circ} \mathrm{C}$, the upper limit for the melting point so that they can be classified as ILs is usually set at $100{ }^{\circ} \mathrm{C}$. Therefore, they can be considered as room-temperature molten salts (Keskin et al., 2007). Such behaviour is obviously related to their chemical composition: ILs are usually composed of bulky and asymmetric organic cations and smaller inorganic or organic anions. This feature makes their lattice energy quite low and, therefore, so does their melting point (Keskin et al., 2007). There are many of them depending on their chemical composition, but almost every IL can be classified, according to its cationic structure, into one of the seven families reported by Torimoto et al. (Torimoto et al., 2010).

Connected to their composition, one of the most interesting properties concerning ILs is their tunability. As a matter of fact, by changing the nature of the constituent ions it is possible to obtain ILs with very different physical and chemical properties (Endes et al., 2008). Theoretically, the combination of cations and anions would lead to a number of ILs as high as $10^{18}$ (Chiappe et al., 2005). In practice, however, this number would be much smaller, but it gives an idea about the broad range of physicochemical properties that can be obtained. For instance, ILs with the same imidazolium cation, [BuMeIm] $]^{1}$, can be either hydrophilic (combined with $\left[\mathrm{BF}_{4}\right]$ or $\left[\mathrm{AlCl}_{4}\right]$ ) or hydrophobic (if the anion is $\left[\mathrm{PF}_{6}\right]$ or $\left[\mathrm{Tf}_{2} \mathrm{~N}\right]$ ) (Torimoto et al., 2010). Here it is worth mentioning the fact that some ILs with magnetic properties have also been prepared by using $\left[\mathrm{FeCl}_{4}\right]$ anions. Tunability is especially interesting in the case of MFs, since it would allow synthesizing tailor-made MFs specifically designed for every single application.

Nevertheless, there are two common features for many of them that are rather interesting from the technological viewpoint. These are their negligible vapour pressure and flammability, at least when compared to those of conventional solvents such as volatile organic compounds (VOCs). Both characteristics ensure, first of all, thermal stability and vacuum resistance and, what is more, an insignificant contribution to air pollution. As a matter of fact, they have been labelled as "green solvents" due to their negligible impact on atmospheric pollution (Keskin et al., 2007; Torimoto et al., 2010). Finally, it is possible to recycle them, which would contribute to reduce their release to the aquatic environment and lead to more efficient and economic industrial processes (Haerens et al., 2010; Wu et al., 2009).

\subsection{Ionic liquid applications}

Since the academic world began to become interested in ILs in the 1990s, the number of scientific articles and patents related to the topic has incredibly increased. For example, in 2000 about 100 patent applications had been reported, while in 2004 this number increased to 800 (Keskin et al., 2007). Nowadays, about 28200 patents related to ILs can be described.

\footnotetext{
1 See apendix (section 7) for nomenclature abbreviations.
} 
Some applications arise from the fact that many ILs are powerful solvents, able to dissolve salts, fats, proteins, amino acids, surfactants or sugars. For instance, [(MeOEt)MeIm] $\left[\mathrm{BF}_{4}\right]$ can dissolve glucose 100 times better than acetone (Park et al., 2001). They can even dissolve crude oil, inks, plastics or DNA. For this reason, they can be used as reaction media for electrochemical and chemical syntheses (Torimoto et al., 2010), giving rise to reaction rates similar or even better than those obtained when using aqueous or organic solvents. Another advantage of using them with this aim is the ease in recovering the resulting products from the reaction medium, especially in those cases in which distillation is not practical, for example, because the reaction products are thermally sensible (Keskin et al., 2007). In addition, it has been reported that ILs can be used as solvents for insoluble bio-related materials like cellulose, which is easily dissolved by strong hydrogen bond-acceptor ILs (like chloride anion-based ones) (Keskin et al., 2007; Torimoto et al., 2010).

Another typical application is their use in gas purification because many gases are soluble in ILs (for example $\mathrm{CO}_{2}$ is highly soluble in [BuMeIm][PF6]) (Anthony et al., 2002). This feature also makes them excellent candidates to be employed in gas absorption applications, together with the fact that gas separation from the IL stream would be very easy (i.e. by simple flash distillation) (Keskin et al., 2007).

Related to materials science, ILs have been widely used in chemical synthesis of nanoparticles, with self-evident advantages like the fact that almost no stabilizing agents that prevent from particle coalescence are needed (requisite almost indispensable in the case of aqueous or organic solvents) (Torimoto et al., 2010). For example, it is possible to synthesize particles for the preparation of MFs such as magnetite (Wang et al., 2009) or cobalt (Behrens et al., 2012) nanoparticles. This would allow an in situ synthesis of the dispersed particles.

As a final point, the preparation of IL-based MFs would allow the use of some of the devices mentioned in section 3 under extreme environmental conditions (ultra-high vacuum or high temperature applications). This would be particularly interesting in the case of space technology such as dampers, dynamic seals or hydrodynamic bearings in gyroscopes for mini-satellites. As a matter of fact, the first MF patent was aimed to prepare controlling fluids for space applications (Papell, 1965).

\section{Ionic liquid-based magnetic fluids}

In this section the research highlights in the field of IL-based MFs are described both in the case of MRFs and FFs. Some details about our group contributions to the field are given too.

\subsection{Ionic-liquid based magnetorheological fluids}

Guerrero-Sánchez et al. (Guerrero-Sánchez et al., 2007) were pioneers in the preparation of IL-based MRFs. More specifically, they prepared several suspensions consisting of either micron- or nanosized magnetite particles dispersed in eight different ILs. The most stable MRF was obtained for a composition of $10 \mathrm{vol} \%$ of magnetite microparticles suspended in 
[BuMeIm][PF6]. As a matter of fact, this MRF only exhibited a sedimentation ratio of 0.95 after two months of preparation. Having prepared a highly stable IL-based MRF was important, but even more important was the fact that colloidal stability was achieved without the addition of any stabilizing agents. The enhancement of stability was attributed to the physical adsorption of the IL ions on magnetite surface (for which they had strong affinity) that gave rise to steric repulsion between the dispersed particles. In the same work, the rheological behaviour of the samples upon magnetic field application was also studied concluding that the MR effect of the IL-based MRFs was comparable to those obtained for conventional MRFs, and high enough for practical purposes.

In a different work, Guerrero-Sánchez et al. (Guerrero-Sánchez et al., 2009) also used ILbased MRFs to study the influence of temperature on their MR behaviour. Here, ILs allowed extending such study to a broader range of temperature than in the case of conventional MRFs, due to their thermal stability and low vapour pressure. Something similar was pointed out by Dodbiba et al. (Dodbiba et al., 2007) who also took advantage of the good performance of ILs at high temperature to study the rheological behaviour upon magnetic field application of a mixture of two types of carbonyl iron powders dispersed in an IL. Therefore, these works make evident that the preparation of IL-based MFs is important from the fundamental point of view too.

Our group has also studied IL-based MRFs, especially for what concerns their stability. With this aim, Gómez-Ramírez et al. (Gómez-Ramírez et al., 2011) prepared IL-based MRFs differing both in the disperse phase material (pure iron and silica-coated iron particles) and in the liquid carrier ([EtMeIm] [EtOSO 3$]$ and ([EtMeIm] [Et $\left.\left.2 \mathrm{OPO}_{3}\right]\right)$. Additionally, conventional MRFs with mineral oil as liquid carrier were also synthesized. The particle volume fraction of all the samples was kept as $10 \mathrm{vol} \%$. The most stable MRF was obtained by the combination of silicacoated iron particles and [EtMeIm] $\left[\mathrm{Et}_{2} \mathrm{OPO}_{3}\right]$; for example, the viscosity of such MRF for shear rates over $100 \mathrm{~s}^{-1}$ almost coincided with the theoretical prediction of Batchelor equation, what means that only two-body hydrodynamic interactions existed between the particles, and, therefore, almost no magnetic aggregates were present. In fact, microscopic observations of the suspensions upon magnetic field application showed that field-induced particle chains had a more regular structure in the case of the most stable MRF (see figure 7). The worst results were obtained for mineral oil-based MRFs in which the particles were strongly aggregated and the resulting structures became more irregular. An intermediate behaviour was found for the suspensions with $[\mathrm{EtMeIm}]\left[\mathrm{EtOSO}_{3}\right]$ as liquid carrier.
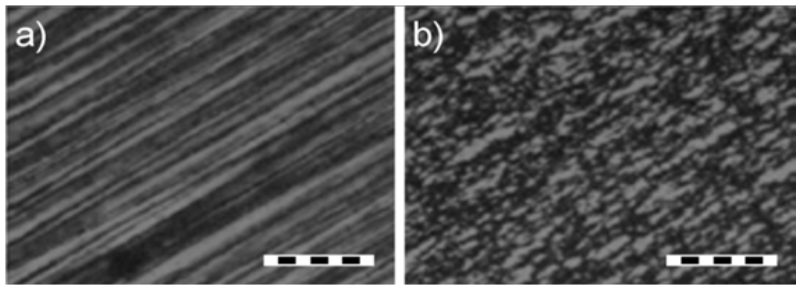

Figure 7. Field-induced particle structures in MRFs consisting of silica-coated iron microparticles dispersed in [EtMeIm] [Et $\left.\mathrm{OPO}_{3}\right]$ (a) and mineral oil (b). Bar length: $200 \mu \mathrm{m}$. 
The improvement of MRF stability when using ILs as carriers, with respect to the ones prepared in mineral oil, was also explained by the adsorption of the IL ions on the surface of the dispersed particles. In a first approximation, such stability appears to be related to the electric conductivity of ILs, which, on its part, is also connected to the anion volume, being the first lower when the latter increases. Accordingly, if the adsorbed ions are larger, the steric repulsion between the particles is stronger too, leading to a decrease in particle aggregation. This is the reason for which a low conductivity-IL like [EtMeIm] $\left[\mathrm{Et}_{2} \mathrm{OPO}_{3}\right]$ gave rise to better results than the high conductivity-one [EtMeIm] [EtOSO 3 . The explanation for the higher stability in the case of MRFs consisting of silica-coated iron particles was the interaction between silica and the constituent IL ions. In fact, the wettability of both ILs was better in the case of silica-coated iron than for bare iron (Gómez-Ramírez et al. 2011).

The direct consequence of the improved stability of IL-based MRFs is a simpler MRF formulation, since no stabilizing additives are needed. This makes easier their preparation at larger scale given that no additional mixing steps are needed, for example. Therefore, stable MRFs with adequate MR response that can be subjected to ultra-high vacuum or very high/low temperatures are obtained, just by using ILs as carrier fluids.

\subsection{Ionic-liquid based ferrofluids}

As it has been pointed out above, one of the advantages of using ILs as carriers is the stability that the physical adsorption of their constituent ions provides with. In the case of MRFs it has been seen that this improvement may be good enough for practical purposes. But what happens in the case of FFs that, by definition, need to be ultrastable? What are the mechanisms involved in the stabilization of IL-based FFs?

The first reference to IL-based FFs appeared in 2009, when Oliveira et al. prepared suspensions of bare maghemite $\left(\gamma-\mathrm{Fe}_{2} \mathrm{O}_{3}\right)$ and cobalt ferrite $\left(\mathrm{CoFe}_{2} \mathrm{O}_{4}\right)$ nanoparticles dispersed in [BuMeIm] $\left.\mathrm{BF}_{4}\right]$ (Oliveira et al., 2009). Such suspensions were said to be stable in the presence of an external magnetic field, even without the addition of stabilizing agents. The authors attributed such stability to the formation of a semi-organized protective layer by the IL ions around the dispersed particles. Nevertheless, when water was added to the suspensions, they became completely unstable, apparently due to the destruction of such a protective layer. The authors also tried to prepare suspensions using hydrophobic ILs (namely $[\mathrm{BuMeIm}]\left[\mathrm{PF}_{6}\right]$ and $[\mathrm{BuMeIm}]\left[\mathrm{Tf}_{2} \mathrm{~N}\right]$ ), but these suspensions were not stable at all.

Jain et al. (Jain et al., 2011) also attempted to synthesize IL-based FFs using maghemite nanoparticles, but with particle concentrations much higher than in the case of Oliveira et al. They succeed when using [EtMeIm][Ac] and [EtMeIm][SCN] as carriers, without needing any stabilizers, but they did not in the case of the protic IL $[\mathrm{EtN}]\left[\mathrm{NO}_{3}\right]$ and [BuMeIm] $\left[\mathrm{BF}_{4}\right]$. In the latter case, they failed even at lower concentrations in contrast with the results of Oliveira et al. As a matter of fact, there is still some controversy related to the stabilization of bare magnetic nanoparticles in IL media, although the stabilization provided by the IL ions has been pointed out to work quite well for metal nanoparticles in general (Pârvulescu et al., 2007). This is the case of positively charged Pt nanoparticles dispersed in [BuMeIm] $\left[\mathrm{BF}_{4}\right]$ and 
[BuMeIm][PF6], for example (Scheeren et al., 2006). However, in some other cases it is not high enough, and additional stabilization is needed (Pârvulescu et al., 2007; Torimoto et al., 2010).

In most of the suspensions mentioned above, the material for the dispersed phase is maghemite. At this point it is important to mention that both Guerrero-Sánchez et al. (Guerrero-Sánchez et al., 2007) and Oliveira et al. (Oliveira et al., 2009) failed to synthesize ultrastable IL-based suspensions when using magnetite $\left(\mathrm{Fe}_{3} \mathrm{O}_{4}\right)$ nanoparticles. [BuMeIm] $\left[\mathrm{BF}_{4}\right]$ that, according to Oliveira et al., had given rise to good results for maghemite and cobalt ferrite, did not work at all in the case of magnetite. This could be due to the fact that magnetic interaction between magnetite nanoparticles is higher than in the case of maghemite ones, since the saturation magnetization of the first $\left(90 \mathrm{Am}^{2} \mathrm{~kg}^{-1}\right)$ is higher than the one of the latter $\left(80 \mathrm{Am}^{2} \mathrm{~kg}^{-1}\right)$. Given that both iron oxide surfaces must be composed of the same $\mathrm{Fe}-\mathrm{OH}$ groups, the formation of the protective layer around magnetite nanoparticles could have taken place, but probably was not protective enough to overcome the magnetic attraction between the particles. An additional stabilization mechanism seemed to be needed.

In 2011 our group reported a true IL-based magnetite FF (Rodríguez-Arco et al., 2011a). In this work, emphasis was made in the fact that only strong steric repulsion was useful to obtain an ultrastable suspension of magnetite in [EtMeIm] [EtOSO 3 ]. This was achieved by adsorbing surfactants with long tails on the surface of magnetite nanoparticles that gave rise to such strong steric repulsion (more details about this IL-based FF are given below). In fact, we also tried to prepare suspensions of bare magnetite nanoparticles in the same IL and failed. Later, something similar was pointed out by Jain et al., who observed that bare maghemite nanoparticles were unstable in the protic IL [EtN][NO 3$]$, but they became highly stable (even at highly particle concentration) when coated by a layer of short acrylic-acid- $b$ acrylamide copolymer (Jain et al., 2012). As a result, steric repulsion seems to be the best stabilization mechanism for the preparation of IL-based FFs.

In view of the results presented above a question may arise: how can we explain that in some cases stabilization is ensured without the addition of stabilizing agents (i.e. surfactants) while in others it is strictly necessary? Here, it is particularly interesting to mention the work by Ueno et al. in which they prepared suspensions of bare and PMMAgrafted silica particles dispersed in imidazolium-based ILs (Ueno et al., 2008). Given that ILs are non-volatile, the in situ behaviour of these suspensions was studied by means of transmission electron microscopy (i.e. no escapes of the liquid to the vacuum system took place). They observed that strong aggregation, which appeared for bare silica particles, was almost absent in the case of PMMA-grafted ones (figure 8). They concluded that electrostatic repulsion, that was the only possible stabilization mechanism in the case of bare silica, did not work. They attributed this to the screening effect of the high ionic atmosphere surrounding the particles, since ILs are actually room-temperature molten salts. They also corroborated such affirmation by estimating the interaction potential energy between the dispersed particles and checking that the electrostatic repulsion component was negligible when compared with the steric repulsion one (Ueno et al., 2008). 


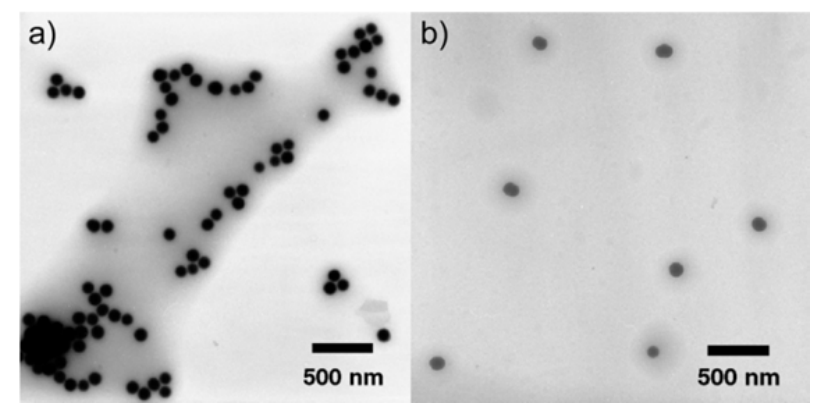

Figure 8. In situ TEM picture of dilute suspensions of bare (a) and PMMA-grafted (b) silica particles in [EtMeIm][Tf 2 N]. Reprinted (adapted) with permission from (Ueno et al., 2008). Copyright (2008) American Chemical Society.

If the results of Ueno et al. (Ueno et al., 2008, 2009) are taken into account, there are two points that seem clear: (i) electrostatic repulsion does not work in IL media because of the highly ionic environment; (ii) only steric repulsion gives rise to long-term stability. On its part, the protective layers formed by the adsorption of the IL ions (i.e. physically or by coordination compounds) could provide with steric repulsion. However, in some cases (as for magnetite suspensions), it is not strong enough to ensure long-term stability and other additives (i.e. surfactants) have to be used.

As it has been pointed out above, our group succeeded in the preparation of true magnetite IL-based FFs. This was only achieved when the surface of the particles was coated with some stabilizing agents, but not all additives worked (Rodríguez-Arco et al., 2011b). For example, citric acid, that had been previously used to stabilize water-based FFs, did not work properly in [EtMeIm][EtOSO 3 , since the samples consisting of citric acid-coated magnetite slightly settled when subjected to a magnetic field gradient of $10 \mathrm{mTmm}^{-1}$, and gave rise to strong phase separation after $5 \mathrm{~min}$ of centrifugation at $8000 \mathrm{~g}$. This was due to the fact that citric acid molecule is very short and, therefore, the resulting steric repulsion was quite weak. As a consequence, it seems that only long enough molecules should be used.

In addition, compatibility between the tails of the surfactant and every particular IL must exist. For example, if magnetite particles were just coated with a single layer of oleic acid, the resulting suspension was as unstable as those consisting of bare magnetite. Nevertheless, if a second layer of oleic acid molecules was formed when an excess of oleic acid was added, a true IL-based FF was obtained. The ultrastability of such FF was accurately proved, since it did not settle either upon magnetic field gradient application or after 30 min of centrifugation at $8000 \mathrm{~g}$. The differences between both particle coatings can be easily observed in figure 9 .

It can be seen that the tails facing the IL in figure 9a are non-polar, while the situation is just the opposite when the oleic acid-double layer is formed (figure 9b). In the first case, there is not compatibility between the surfactant tails and the IL (highly polar), whereas in the second one there is. As it has been previously pointed out, Jain et al. (Jain et al., 2012) were 
able to stabilize maghemite nanoparticles in $[\mathrm{EtN}]\left[\mathrm{NO}_{3}\right]$ by coating them by a layer of short acrylic-acid- $b$-acrylamide copolymer; however, they were not in the case of [BuMeIm] $\left[\mathrm{BF}_{4}\right]$ and [EtMeIm][SCN] just because the polyacrylamide block of the acrylic-acid- $b$-acrylamide copolymer is not soluble in these ILs, and therefore, no compatibility exists. Therefore, as it has been said above, the best way to ensure long-term stability is the use of surfactants with long enough, carrier liquid-compatible tails adsorbed on the surface of magnetite nanoparticles.
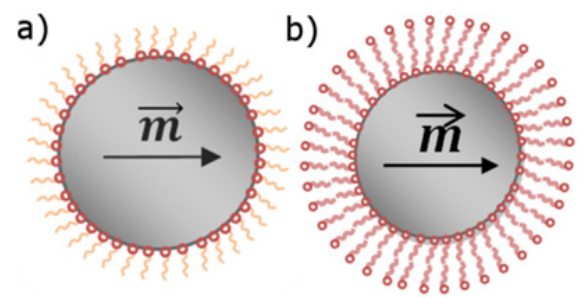

Figure 9. Monolayer of oleic acid molecules adsorbed on the surface of magnetite nanoparticles (a). Oleic acid-double layer formed when adding an excess of oleic acid (b).

Finally, some results about the rheological behaviour upon magnetic field of the samples mentioned above are shown (Rodríguez-Arco et al., 2011b). In particular, figure 10 shows the yield stress obtained for all of them.

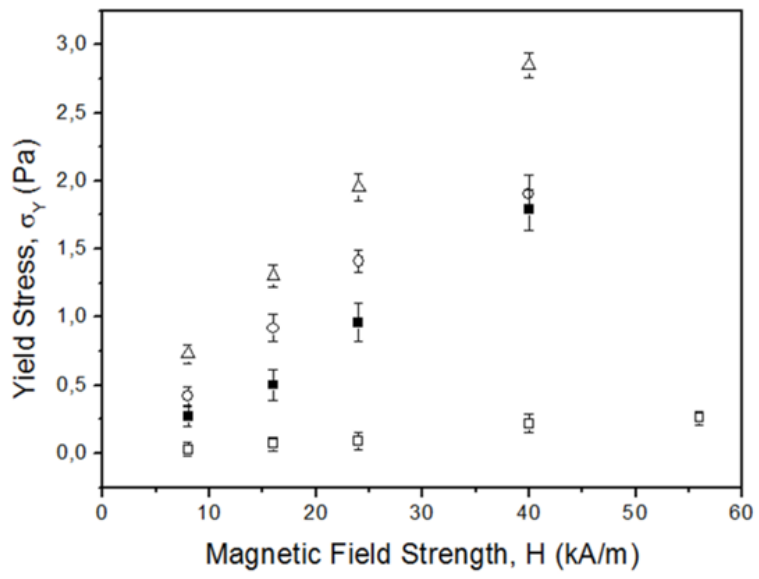

Figure 10. Yield stress for IL-based suspensions consisting of bare (triangles), citric acid-coated (circles) and oleic acid-double layer-coated (squares) magnetite. Full and open squares correspond to this latter sample before and after centrifugation respectively (see text).

With regards to the results presented in figure 10 it is important to remind that, theoretically, a true FF should not display considerable yield stresses, since its response to magnetic field is too weak for this to happen. However, in the case of the suspension of bare magnetite, the yield stress is quite high, likely due to the strong particle aggregation, and therefore, to the formation of field-induced structures by the aggregates. When the particles 
are coated by citric acid, the aggregation degree decreases, and so does the yield stress. In the case of the oleic acid-double layer two different situations appear. The first one corresponds to the original sample, which displays similar values (a little bit lower) than the sample of citric acid-coated magnetite. However, when this sample is subjected to centrifugation, although the supernatant itself is still magnetic, the yield stress of the supernatant becomes negligible. This can be explained because particle aggregates that could be present in the original sample were removed by centrifugation. Similar results were found for the MV effect (Rodríguez-Arco et al., 2011b). In fact, the MV response of these new IL-based MFs was theoretically fitted by a model that was originally proposed by Zubarev et al. (Zubarev et al., 2005) for oil-based FFs, showing that the MV effect increases with both the volume of the largest particles and their concentration and when the distance between the magnetic cores of the particles decreases.

In conclusion, it could be said that much work is being done in the field of IL-based FFs. Thanks to such studies our understanding of the phenomena involving them (i.e. stability) is improving, and it is very likely that better IL-based FFs will be prepared in the future. However, more applied studies are needed, which, for example, analyze their real performance in technological devices like those of section 3.

\section{Future challenges}

Many of the future challenges in the field of IL-based MFs are related to ILs in general. One of the most important disadvantages of ILs, for instance, is their cost, that can be much higher than that of conventional organic solvents. However, in some specific applications, it is probably worth using them (i.e. space applications). Nevertheless, their price will decrease if they begin to be produced at a larger scale.

Another disadvantage of ILs when compared with traditional carriers is the lack of enough data about their physicochemical properties and toxicity (Keskin et al., 2007). In the same direction, a thorough analysis of the relationship (e.g. adsorption, wettability) between the particle surface and the constituent ions in MFs would be of the utmost importance, in order to gain a better understanding of the stability mechanisms.

Since ILs could be used to prepare tailor-made MFs for each specific application, it would be interesting to broaden their preparation by changing the IL carrier, the nature of the dispersed phase or its concentration. But before this is accomplished, it is necessary to determine which solid materials (or surfactants in the case of FFs) fit best each particular IL. As a result, deeper studies on the compatibility among all the MF constituents may be needed in the future. Additionally, further research has to be done, not only with regards to the formulation of IL-based MFs, but also to their performance in each particular application. As a consequence, detailed magnetization and magnetorheological studies should be faced too.

The final step for IL-based MFs would need a much more applied study which could result in a number of patents susceptible of being exploited. For example, they should be included 
in prototypes before developing any industrial device. This would allow additional industrial implementation and commercialization of this new breed of MFs. Here it is worth mentioning the work by companies like Ioniqa Technologies ${ }^{\odot}$ that are starting to commercialize different IL-based magnetic smart materials, including MFs, magnetoresponsive elastomers, and magnetic ILs. Therefore, this new type of MFs will pose new questions not only to scientists and engineers, but also to businesspeople.

\section{Conclusions}

In this book chapter an overview of the latest advances and research highlights in the field of MFs has been done, especially concerning the use of room-temperature ILs as fluid carriers. As it has been described, there are numerous MF applications that could benefit from IL features such as low flammability, negligible vapor pressure and tunability. However, although a lot of work has been done, there are still problems that have to be overcome if a wider commercialization of IL-based MFs is desired. Therefore, these new horizons broadened by their potential uses are, at the same time, encouraging and challenging.

\section{Appendix}

Here we list the IL nomenclature abbreviations used in the chapter.

\begin{tabular}{|l|l|l|}
\hline \multirow{4}{*}{ Cations } & {$[$ EtMeIm $]$} & 1-ethyl-3-methylimidazolium \\
\cline { 2 - 3 } & {$[\mathrm{BuMeIm}]$} & 1-butyl-3-methylimidazolium \\
\cline { 2 - 3 } & {$[(\mathrm{MeOEt}) \mathrm{MeIm}]$} & 1-methoxyethyl-3-methylimidazolium \\
\cline { 2 - 3 } & {$[\mathrm{EtN}]$} & Ethylammonium \\
\hline \multirow{5}{*}{ Anions } & {$[\mathrm{Ac}]$} & Acetate \\
\cline { 2 - 3 } & {$\left[\mathrm{Tf}_{2} \mathrm{~N}\right]$} & Bis(trifluoromethylsulfonyl) imide \\
\cline { 2 - 3 } & {$\left[\mathrm{Et}_{2} \mathrm{OPO}_{3}\right]$} & Diethylphosphate \\
\cline { 2 - 3 } & {$\left[\mathrm{EtOSO}_{3}\right]$} & Ethylsulphate \\
\cline { 2 - 3 } & {$\left[\mathrm{PF}_{6}\right]$} & Hexafluoroborate \\
\cline { 2 - 3 } & {$\left[\mathrm{NO}_{3}\right]$} & Nitrate \\
\cline { 2 - 3 } & {$\left[\mathrm{AlCl}_{4}\right]$} & Tetrachloroaluminate \\
\cline { 2 - 3 } & {$\left[\mathrm{FeCl}_{4}\right]$} & Tetrachloroferrate \\
\cline { 2 - 3 } & {$\left[\mathrm{BF}_{4}\right]$} & Tetrafluoroborate \\
\hline
\end{tabular}

Table 1.

\section{Author details}

Laura Rodríguez-Arco, Ana Gómez-Ramírez, Juan D.G. Durán and Modesto T. López-López

University of Granada, Spain 


\section{Acknowledgement}

This work is supported by projects P08-FQM-3993 and P09-FQM-4787 (Junta de Andalucía, Spain) and FIS2009-07321 (MICINN, Spain). L.R.-A. and M.T.L.-L. also acknowledge financial support by the Secretaría de Estado de Educación, Formación Profesional y Universidades (MECD, Spain) through its FPU Program and University of Granada (Spain), respectively.

\section{References}

Anthony, J.L.; Maginn, E.J. \& Brennecke, J.F. (2002). Solubilities and Thermodynamic Properties of Gases in the Ionic Liquid 1-n-butyl-3-methylimidazolium hexafluorophosphate. The Journal of Physical Chemistry B, Vol. 106, No. 29, (July 2002), pp. 7315-7320, ISSN 1520-6106

Behrens, S. \& Essig, S. (2012). A Facile Procedure for Magnetic Fluids Using Room Temperature Ionic Liquids. Journal of Materials Chemistry, Vol. 22, No. 9, (March 2012), pp. 3811-3816, ISSN 0959-9428

Bossis G.; Volkova, O.; Lacis, S. \& Meunier, A. (2002). Magnetorheology: Fluids, Structures and Rheology, In: Ferrofluids. Magnetically Controllable Fluids and Their Applications, S. Odenbach, pp. 186-230, Lecture Notes in Physics (Springer-Verlag), ISBN 3-540-43978-1, Berlin, Germany

Cao, L.; Park, H. S.; Dodbiba, G. \& Fujita, T. (2010). Synthesis of an Ionic Liquid-based Magnetorheological Fluid Dispersing $\mathrm{Fe}_{44} \mathrm{Nb}_{3} \mathrm{~V}_{4} \mathrm{~B} 9$ Nanocrystalline Powders. International Journal of Modern Physics B, Vol. 24, No. 10, (April 2010), pp. 1227-1234, ISSN 0217-9792

Carlson, J.D.; Catanzarite, D.M. \& St. Clair, K.A. (1996). Properties and Applications of Commercial Magnetorheological Fluids. International Journal of Modern Physics B, Vol. 10, No. 23-24, (October 1996), pp. 2857-2865, ISSN 0217-9792

Charles, S.W. (2002). Magnetic Fluids. Ferrofluids, In: Ferrofluids. Magnetically Controllable Fluids and Their Applications, S. Odenbach, pp. 3-18, Lecture Notes in Physics (SpringerVerlag), ISBN 3-540-43978-1, Berlin, Germany

Chiappe, C. \& Pieraccini, D. (2005). Ionic liquids: Solvent Properties and Organic Reactivity. Journal of Physical Organic Chemistry, Vol. 18, No. 4, (April 2005), pp.275-297, ISSN 10991395

Dodbiba, G.; Park, H. S.; Okaya, K. \& Fujita, T. (2008). Investigating Magnetorheological Properties of a Mixture of Two Types of Carbonyl Iron Powders Suspended in an Ionic Liquid. Journal of Magnetism and Magnetic Materials, Vol. 320, No. 7, (April 2008), pp. 1322-1327, ISSN 0304-8853

de Gans, B.J.; Blom, C.; Philipse, A.P. \& Mellema, J. (1991). Linear Viscoelasticity of an Inverse Ferrofluid. Physical Review E, Vol. 60, No. 4, (October 1999), pp. 4518-4527, ISSN 1539-3755 
Durán, J.D.G.; Arias, J.L.; Gallardo, V. \& Delgado, A.V. (2007). Magnetic Colloids as Drug Vehicles. Journal of Pharmaceutical Sciences, Vol. 97, No. 8, (December 2007), pp. 29482983, ISSN 1520-6017

de Vicente, J.; López-López, M.T.; González-Caballero, F. \& Durán, J.D.G. (2003). Rheological Study of the Stabilization of Magnetizable Colloidal Suspensions by Addition of Silica Nanoparticles. Journal of Rheology, Vol. 47, No. 5, (November 2003), pp. 1093-1109, ISSN 0148-6055

Endes, F.; Abbot, A.P. \& MacFarlane, D.R. (2008). Electrodeposition from Ionic Liquids, WileyVCH, ISBN 978-3-527-31565-9, New York, USA

Guerrero-Sánchez, C.; Lara-Ceniceros, T.; Jiménez-Regalado, E., Raşa, M. \& Schubert, U.S. (2007). Magnetorheological Fluids Based on Ionic Liquids. Advanced Materials, Vol. 19, No. 13, (July 2007), pp.1740-1747, ISSN 1521-4095

Guerrero-Sánchez, C.; Ortiz-Alvarado, A. \& Schubert, U.S. (2009). Temperature Effect on the Magneto-Rheological Behavior of Magnetite Particles Dispersed in an Ionic Liquid. Journal of Physics: Conference Series, Vol. 149, pp. 012052-012056, ISSN 1742-6588

Haerens, K.; Van Deuren, S.; Matthijs, E. \& Van der Bruggen, B. (2010). Challenges for recycling ionic liquids by using pressure driven membrane processes. Green Chemistry, Vol. 12, No. 12, (December 2010), pp. 2182-2188, ISSN 1463-9262

Herpin, A. (1968). Théorie du Magnétisme, Institut National des Sciences et Techniques Nucléaires, PUF, Saclay-Paris, France

Jain, N; Zhang, X.; Hawkett, B.S. \& Warr, G.G. (2011). Stable and Water-Tolerant Ionic Liquid Ferrofluids. Applied Materials E Interfaces, Vol. 3, No. 3, (March 2011), pp. 662667, ISSN 1944-8244

Jeong, U; Teng, X.W.; Wang, Y.; Yang, H. \& Xia, Y.N. (2007). Superparamagnetic Colloids: Controlled Synthesis and Niche Applications. Advanced Materials, Vol. 19, No. 1, (January 2007), pp. 33-60, ISSN 1521-4095

Jolly, M.R.; Bender, J.W. \& Carlson, J.D. (1999). Properties and Applications of Commercial Magnetorheological Fluids. Journal of Intelligent Material Systems and Structures, Vol. 10, No. 1., (January 1999), pp. 5-13, ISSN 1530-8138

Keskin, S.; Kayrak-Talay, D.; Akman, U. \& Ortaçsu, O. (2007). A Review of Ionic Liquids Towards Supercritical Fluid Applications. Journal of Supercritical Fluids, Vol. 43, No. 1, (November 2007), pp. 150-180, ISSN 0896-8446

Klingenberg, D.J. (2001). Magnetorheology: Applications and Challenges. AlChhE JournalK, Vol. 47, No. 2, (February 2001), pp. 246-249, ISSN 1547-5905

Kuzhir, P.; López-López, M.T. \& Bossis, G. Magnetorheology of Fiber Suspensions. II. Theory. (2009). Journal of Rheology, Vol. 53, No. 1, (January 2009), pp. 127-151, ISSN 01486055

López-López, M.T.; de Vicente, J.; González-Caballero, F. \& Durán, J.D.G. (2005a). Stability of Magnetizable Colloidal Suspensions by Addition of Oleic Acid and Silica Nanoparticles. Colloids and Surfaces A: Physicochemical and Engineering Aspects, Vol. 264, No.1-3, (August 2005), pp. 75-81, ISSN 0927-7757

López-López, M.T.; de Vicente, J.; Bossis, G.; González-Caballero, F. \& Durán, J.D.G. (2005b). 
Preparation of Stable Magnetorheological Fluids Based on Extremely Bimodal Ironmagnetite Suspensions. Journal of Materials Research, Vol. 20, No. 4, (April 2005), pp. 874881, ISSN 0884-2914

López-López, M.T.; Kuzhir, P.; Lacis, S.; González-Caballero, F.; Durán, J.D.G. \& Bossis, G. (2006). Magnetorheology for Suspensions of Solid Particles Dispersed in Ferrofluids. Journal of Physics: Condensed Matter, Vol. 18, No. 38, (September 2006), pp. S2803-S2813, ISSN 0953-8984

López-López, M.T.; Vertelov, G.; Bossis, G.; Kuzhir, P. \& Durán, J.D.G. (2007). New Magnetorheological Fluids Based on Magnetic Fibers. Journal of Materials Chemistry, Vol. 17, No. 36, (September 2007), pp. 3839-3844, ISSN 0959-9428

López-López, M.T.; Gómez-Ramírez, A.; Durán, J.D.G. \& González-Caballero, F. (2008). Preparation and Characterization of Iron-Based Magnetorheological Fluids Stabilized by Addition of Organoclay Particles. Langmuir, Vol. 24, No. 14, (June 2008), pp. 70767084, ISSN 0743-7463

López-López, M.T.; Kuzhir, P. \& Bossis, G. (2009). Magnetorheology of Fiber Suspensions. I. Experimental. Journal of Rheology, Vol. 53, No. 1, (January 2009), pp. 115-126, ISSN 01486055

López-López, M.T.; Zubarev, A. \& Bossis, G. (2010). Repulsive Force Between Two Attractive Dipoles, Mediated by Nanoparticles Inside a Ferrofluid. Soft Matter, Vol. 6, No. 18, (September 2010), pp. 4346-4349, ISSN 1744-683X

López-López, M.T.; Gómez-Ramírez, A.; Rodríguez-Arco, L.; Durán, J.D.G., Iskakova, L. \& Zubarev, A. (2012). Colloids on the Frontier of Ferrofluids. Rheological Properties. Langmuir, in press, DOI: 10.1021/la204112w, ISSN 0743-7463

Odenbach, S. \& Thurm S. (2002). Magnetoviscous Effects in Ferrofluids, Lecture Notes in Physics (Springer-Verlag), ISBN 3-540-43068-1, Berlin Germany

Oliveira, F.C.C.; Rossi, L.M.; Jardim, R.F. \& Rubim, J.C. (2009). Magnetic Fluids Based on $\gamma$ $\mathrm{Fe}_{2} \mathrm{O}_{3}$ and $\mathrm{CoFe}_{2} \mathrm{O}_{4}$ Nanoparticles Dispersed in Ionic Liquids. The Journal of Physical Chemistry C, Vol. 113, No. 20, (May 2009), pp. 8566-8572, ISSN 1932-7447

Papell, S.S. (1965). US Patent Specification 3215572.

Park, S. \& Kazlauskas, R.J. (2001). Improved Preparation and Use of Room-temperature Ionic Liquids in Lipase-catalyzed Enantio- and Regioselective Acylations. The Journal of Organic Chemistry, Vol. 66, No. 25, (December 2001), pp. 8395-8401, ISSN 0022-3263

Park, B.J.; Fang F.F. \& Choi, H.J. (2010). Magnetorheology: Materials and Application. Soft Matter, Vol. 6, No. 21, (November 2010), pp. 5246-5253, ISSN 1744-683X

Pârvulescu, V.I. \& Hardacre, C. Catalysis in Ionic Liquids. (2007). Chemical Reviews, Vol. 107, No. 6, (June 2007), pp. 2615-2665, ISSN 0009-2665

Popplewell, J. (1984). Technological Applications of Ferrofluids. Physics in Technology, Vol. 15, No. 3, (May 1984), pp. 150-156, ISSN 0305-4624

Ramos, J.; Klingenberg, D.J., Hidalgo-Álvarez, R. \& de Vicente, J. (2011). Steady Shear Magnetorheology of Inverse Ferrofluids. Journal of Rheology, Vol. 55, No. 1, (January 2011), pp. 127-152, ISSN 0148-6055

Raj, K. \& Moskowitz, R. (1990). Commercial Applications of Ferrofluids. Journal of Magnetism and Magnetic Materials, Vol. 85, No. 1-3, (April 1990), pp. 233-245, ISSN 0304-8853 
Rodríguez-Arco, L.; López-López, M.T.; González-Caballero, F. \& Durán, J.D.G. (2011a). Steric Repulsion as a Way to Achieve the Required Stability for the Preparation of Ionic Liquid-based Ferrofluids. Journal of Colloid and Interface Science, Vol. 357, No. 1, (May 2011), pp. 252-254, ISSN 0021-9797

Rodríguez-Arco, L.; López-López, M.T.; Durán, J.D.G.; Zubarev, A. \& Chirikov, D. (2011b) Stability and Magnetorheological Behaviour of Magnetic Fluids Based on Ionic Liquids. Journal of Physics: Condensed Matter, Vol. 23, No. 45, (November 2011), pp. 455101455116, ISSN 0953-8984

Rosensweig, R.E. (1985). Ferrohydrodynamics, Cambridge University Press, ISBN 0-486-678342, New York, USA

Scheeren, C.W.; Machado, G.; Teixeira, S.R.; Morais, J.; Domingos, J.B. \& Dupont, J. (2006). Synthesis and Characterization of $\operatorname{Pt}(0)$ Nanoparticles in Imidazolium Ionic Liquids. The Journal of Physical Chemistry B, Vol. 116, No. 26, (July 2006), pp. 13011-13020, ISSN 15206106

Shima, P.D. \& Philip, J. (2011). Tuning of Thermal Conductivity and Rheology of Nanofluids Using an External Stimulus. The Journal of Physical Chemistry C, Vol. 115, No. 41, (October 2011), pp. 20097-20104, ISSN 1932-7447

Torimoto, T.; Tsuda, T.; Okazaki, K. \& Kuwabata, S. (2010). New Frontiers in Materials Science Opened by Ionic Liquids. Advanced Materials, Vol. 22, No. 11, (March 2010), pp. 1196-1221, ISSN 1521-4095

Tran, N. \& Webster T.J. (2010). Magnetic Nanoparticles: Biomedical Applications and Challenges. Journal of Materials Chemistry, Vol. 20, No. 40, (October 2010), pp. 8760-8767, ISSN 0959-9428

Ueno, K., Inaba, A., Kondoh, M. \& Watanabe, M. (2008). Colloidal Stability of Bare and Polymer-Grafted Silica Nanoparticles in Ionic Liquids. Langmuir, Vol. 24, No. 10, (May 2008), pp. 5253-5259, ISSN 0743-7463

Ueno, K., Imizumi, S., Hata, K. \& Watanabe, M. (2009). Colloidal Interaction in Ionic Liquids: Effects of Ionic Structures and Surface Chemistry on Rheology of Silica Colloidal Dispersions. Langmuir, Vol. 25, No. 2, (January 2009), pp. 825-831, ISSN 07437463

Wang, Y. \& Yang, H. (2009). Synthesis of Iron Oxide Nanorods and Nanocubes in an Imidazolium Ionic Liquid. Chemical Engineering Journal, Vol. 147, No. 1, (April 2009), pp. 71-78, ISSN 1385-8947

Wu, B.; Liu, W.; Zhang, Y. \& Wang, H. (2009). Do We Understand the Recyclability of Ionic Liquids? Chemistry - A European Journal, Vol. 15, No. 8, (February 2009), pp. 1804-1810, ISSN 1521-3765

Zubarev, A. Yu.; Fleisher, J. \& Odenbach, S. (2005). Towards a theory of dynamical properties of polydisperse magnetic fluids: effect of chain-like aggregates. Physica $A$, Vol. 358, No. 2-4, (December 2005), pp. 475-91, ISSN: 0378-4371 